\title{
Percutaneous Drainage of Splenic Tubercular Abscess: Assessment of Effectiveness and Safety
}

\author{
S. Bellasri ${ }^{1 *}$, B. Slioui ${ }^{1}$, N. Hamoune ${ }^{1}$, H. Baba ${ }^{2}$, A. Raissi ${ }^{3}$, M. Miloudi ${ }^{4}$, A. Mouhsine ${ }^{1}$, E. Atmane ${ }^{1}$
}

${ }^{1}$ Interventional Radiology Department, Avicenne Military Hospital, University CadiAyyad, Marrakech, Morocco

${ }^{2}$ Surgery Department, Avicenne Military Hospital, University CadiAyyad, Marrakech, Morocco

${ }^{3}$ Hematologic department, Avicenne Military Hospital, University CadiAyyad, Marrakech, Morocco

${ }^{4}$ Biological Department, Avicenne Military Hospital, University CadiAyyad, Marrakech, Morocco

DOI: $10.36347 /$ sjmcr.2021.v09i03.009

| Received: 21.02.2021 | Accepted: 04.03.2021 | Published: 10.03.2021

*Corresponding author: Bellasri Salah

Abstract

Case Report

Splenic tubercular abscess is a rare pathologic conditionand mainly affects immunocompromised individuals. Open surgery and antituberculous drugs was the treatment of choice. Mini invasive procedures such us percutaneous catheter drainage is currently an effective and safe alternative to splenectomy. We present a case of a 24-year-old man with recently diagnosis of Hodgkin's lymphoma with good socioeconomic background, who endured fever and left upper abdominal pain; however, computed tomography scan for extension of Hodgkin's lymphoma revealed a large splenic collection with thick rim interpreted as abscess. Percutaneous drainage of the abscess was performed by interventional radiology. Diagnosis of mycobacterium tuberculosis was confirmed by rapid molecular detection using automated GeneXpert MTB. Our case highlights the following: (a) The importance of minimal invasive procedure in health care system, that reduces length hospitalization, surgical complication and increase cost-effectiveness; (b) The safety of percutaneous drainage even in spleen which may scare radiologist.

Keywords: Splenic, tuberculosis, abscess, percutaneous drainage, US guided.

Copyright () 2021 The Author(s): This is an open-access article distributed under the terms of the Creative Commons Attribution 4.0 International License (CC BY-NC 4.0) which permits unrestricted use, distribution, and reproduction in any medium for non-commercial use provided the original author and source are credited.

\section{INTRODUCTION}

Splenic tubercular abscess is an uncommon entity, with a few reported cases [1]. Its frequently observed in immunocompromised patients [2-5]. Usually, it concerns disseminated $\mathrm{TB}$, and primary splenic TB remains uncommon with only few cases published in the literature $[6,7]$.

Because of its wide range of nonspecific presenting symptoms, it is commonly misdiagnosed and imaging play a crucial contribution in early diagnosis. Traditionally surgery and antituberculous therapy was the principal therapeutics. More recently percutaneous imaging guided drainage was adopted as being safe and effective procedure in splenic abscess. [8-12].

We describe a rare case of tubercular splenic abscess in a Hodgkin's lymphoma patient. We report our experience ofpercutaneous drainage of splenic abscess under imaging guidance. We emphasize the efficacy of the procedure and cost-effectiveness, highlighting the safety and rapidity of this management.

\section{Case Presentation}

A 24-year old man with the chief complaints being fever, left upper abdominal pain and left cervical swelling which gradually increased in size and associated pain for 1 month. Had multiple medical visits and multiple courses of antibiotics over this period, with no reduction in symptoms. Physical examination revealed multiple palpable lymph nodes, including left submandibular and level II cervical lymph nodes with the largest one measuring approximately $2 \mathrm{~cm} \times 3 \mathrm{~cm}$ in size, associated with smooth, firm, nontender mass in the left hypochondriac region.

Ultrasonography (US) revealed splenomegaly with a large splenic hypoechoic lesion with after enhancement suggestive cystic lesion within the spleen. The wall of the lesion was regular and thick. In neck, US confirmed the presence of larges homogenous lymph nodes.

Computed tomography CT scan was performed before and after intravenous contrast administration, and explained a large well defined 
$8 \times 7 \mathrm{~cm}$ sized water attenuation lesion within the spleen (Fig-1). Two small adjacent cystic lesions were observed. Labs were remarkable for leukocytosis of $17.6 / \mathrm{mm} 3$, CRP level at $250 \mathrm{mg} / \mathrm{L}$.

Percutaneous drainage was performed under sonographic guidance, using a pigtail catheter 9F (Boston Scientific, Quincy, MA, USA). Analysis of pus was sterile but rapid molecular detection was positive for mycobacterium tuberculosis by the automated GeneXpert MTB system.

Simultaneous biopsy of cervical lymphnodes by automated core needle biopsy using an $18 \mathrm{G}$ device $\left(\right.$ Monopty ${ }^{\mathrm{TM}}$ ), revealed a histologic pattern of Hodgkin's lymphoma.

Control CT of splenic abscess realised after 48 h confirmed successful complete discharge of collection (Fig-2).

The patient was subsequently started on fourdrug antitubercular protocol. He was referred to haematologist for management of his Hodgkin's lymphoma.

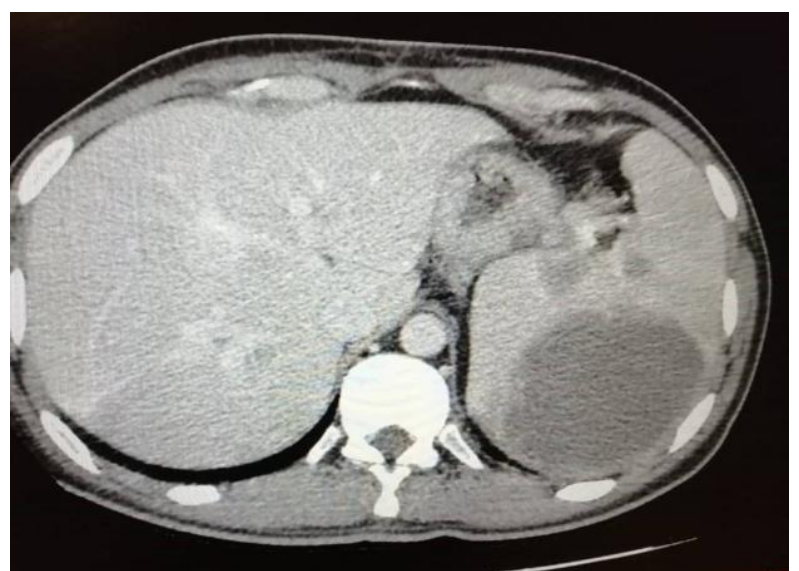

Fig-1: Axial of upper abdominal region CT after intravenous contrast injection, revealing large cystic lesion in spleen, associated with two similar perihilar collections, and rim enhancement

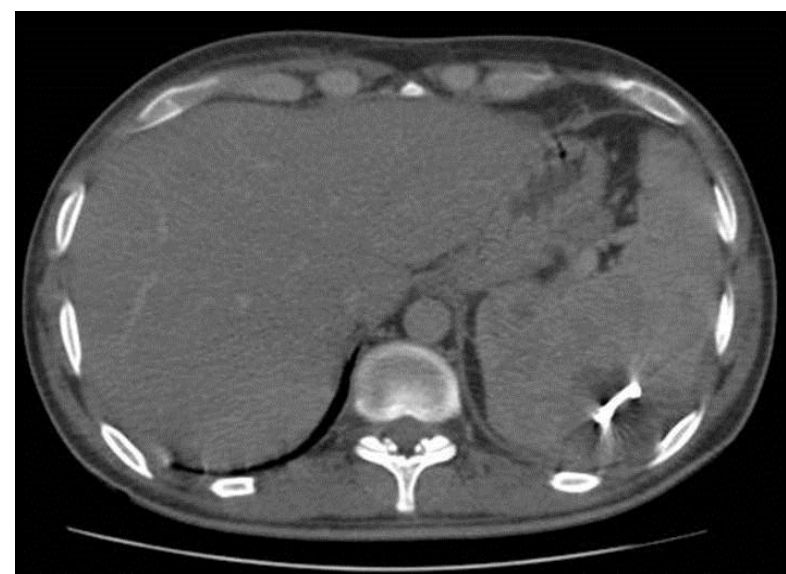

Fig-2: Axial CT after 48h drainage using catheter pigtail, with complete discharge of splenic abscess

\section{DISCUSSION}

Tuberculosis is a major health system threats all over the world and pulmonary tuberculosis is the most common manifestation. Extra pulmonary disease accounts for almost 15-20\% of all tuberculosis [7].

Primary splenic tuberculosis is rare, which usualy affects immunocompromised patients [13]. Simultaneous Hodgkin's lymphoma diagnosis in our case, explained his underlying immunocompromised status. To our knowledge this is de first report of this pathologic association. Splenic abscess is an extremely rare condition with an incidence in autopsy studies of $0.14-0.7 \%$ [14]

Clinical complaints may be non-specific, and diagnosis of splenic TB is often delayed. Fever, weight loss, anorexia, and abdominal pain are the chief symptoms. Imaging play a major role in diagnosis and guidance of puncture or drainage treatment option [15].

It helps to assess and characterize the lesion, identify a suitable site for aspiration and in percutaneous drainage.

On ultrasonography (US), hypoechoic lesions represent tuberculomas, which may be multiple and necrotic or hemorrhagic; whereas, anechoic lesions with thick wall are more suggestive of abscess [16]. On CT, splenic TB has a characteristic appearance of solitary or multiple hypodense nodular areas. Water condensation with thick wall enhancement indicates abscess formation [17].

Biological confirmation is still necessary to confirmate tuberculous origine of the abscess. Direct analysis and culture were the traditional tools, with high rate of false negative results. More recently, rapid molecular detection using automated GeneXpert MTB system has a combined sensitivity and specificity of $77.3 \%$ and $98.2 \%$, respectively, for extrapulmonary TB [18].

Classically patients with tubercular abscess, were treated by splenectomy and antitubercular protocol. In our case, because of the risk of surgery and young age of the patient, percutaneous drainage of the abscess was carried out with successful and safe installation of a catheter during 48h. Subsequently antitubercular protocol was prescribed. After ablation of catheter, patient was referred to haematologist for management of his associated Hodgkin's lymphoma.

\section{CONCLUSION}

TB should be considered as differential diagnosis in case of isolated splenic abscesses in immunocompromised patients from endemic areas. Clinical manifestations remain non-specific and imaging is crucial for prompt and faster diagnosis. Also, 
imaging allows percutaneous puncture and drainage guidance. Mini invasive procedure offer many advantages and is obviously preferred to surgical splenectomy.

\section{REFERENCES}

1. Agarwala S, Bhatnagar V, Mitra DK. Primary tubercular abscess of the spleen. J Pediatr Surg.1992; 27:1580-1.

2. Ooi LL, Leong SS. Splenic abscesses from 1987 to 1995 . Am J Surg. 1997; 174:87-93.

3. de Bree E, Tsiftsis D, Christodoulakis M, Harocopos G, Schoretsanitis G, Melissas J. Splenic abscess: a diagnostic and therapeutic challenge. Acta Chir Belg. 1998;98:199-202.

4. Smyrniotis V, Kehagias D, Voros D, Fotopoulos A, Lambrou A, Kostopanagiotou G. Splenic abscess. An old disease with new interest. Dig Surg. 2000;17:354-7.

5. Chang KC, Chuah SK, Changchien CS, Tsai TL, $\mathrm{Lu}$ SN, Chiu YC. Clinical characteristics and prognostic factors of splenicabscess: a review of 67 cases in a single medical center of Taiwan. World J Gastroenterol. 2006;12:460-4.

6. Kumar S, Pai AG, Tungenwar PN. Isolated primary tuberculosis of spleen-A rare entity in the immuno-competent patient. Int J Surg Case Rep. 2017;30:93-6.

7. Hamizah R, Rohana AG, Anwar SA, et al. Splenic tuberculosis presenting as pyrexia of unknown origin. Med J Malaysia. 2007;62:70-1.

8. Liu KY, Shyr YM, Su CH, Wu CW. Splenic abscess: a changing trend in treatment. S Afr J Surg. 2000;38:55-57.
9. Chou YH, Hsu CC, Tiu CM, Chang T. Splenic abscess: sonographic diagnosis and percutaneous drainage or aspiration. Gastrointest Radiol. 1992;17:262-266.

10. Hadas-Halpren I, Hiller N, Dolberg M. Percutaneous drainage of splenic abscesses: an effective and safe procedure. $\mathrm{Br} \mathrm{J}$ Radiol. 1992;65:968-970.

11. Green BT. Splenic abscess: report of six cases and review of the literature. Am Surg. 2001;67:80-85.

12. Chou YH, Tiu CM, Chiou HJ, Hsu CC, Chiang $\mathrm{JH}, \mathrm{Yu} \mathrm{C}$. Ultrasound-guided interventional procedures in splenic abscesses. Eur J Radiol. 1998;28:167-170.

13. Pramesh CS, Tamhankar AP, Rege SA, Shah SR. Splenic tuberculosis and HIV-1 infection. Lancet. 2002;359(9303):353.

14. Paris S, Weiss Sm, ayers WH Jr, Clarke le. Splenic abscess. am Surg. 1994 may;60(5):358361.

15. Singh B, Ramdial PK, Royeppen e, moodley J, Chetty R. Isolated splenic tuberculosis. Trop doct. 2005 Jan;35(1):48-49.

16. Sharma SK, Smith-Rohrberg D, Tahir M, Mohan A, Seith A. Radiological manifestations of splenic tuberculosis: A 23-patient case series from India. Indian J Med Res. 2007;125:669-78.

17. Xie R, Zhou X, Chen J. CT in diagnosis of tuberculosis of liver and spleen. ZhonguaJie $\mathrm{He}$ He Hu Xi ZaZhi. 1999;22:237-8.

18. Hillemann d, Rüsch-Gerdes S, Boehme C, Richter E. Rapid molecular detection of extrapulmonary tuberculosis by the automated GeneXpertmTB/RIF system. J Clinmicrobiol. 2011 apr;49(4):1202-1205. 